\title{
LA UNIDAD DE OPUESTOS EN LEIBNIZ
}

\section{THE UNITY OF OPPOSITES}

\author{
Francisco Javier Luna Leal ${ }^{1}$ \\ Facultad de Filosofía y Letras, UNAM (México)
}

Recibido: 6-9-2014

Aceptado: 15-3-2015

Resumen: El desarrollo del cálculo infinitesimal le permitió a Leibniz establecer un puente entre entidades que anteriormente se consideraban opuestas y mostrar la armonía subyacente. Con esa experiencia Leibniz trató de conciliar entre la individualidad y el continuo, estableciendo una armonía entre las sustancias individuales y el universo infinito.

Palabras-clave: cálculo; sustancia; unidad; opuestos; infinito.

\begin{abstract}
The development of calculus allowed Leibniz to build up a bridge between entities previously considered opposite and show the underlying harmony. With that experience Leibniz tried to reconcile the individuality and the continuity, establishing a harmony between individual substances and the infinite universe.
\end{abstract}

Key-words: calculus; substance; unity; opposites; infinity.

[1] (lechuza@unam.mx) Doctor en Historia por la Universidad Nacional Autónoma de México en la línea de teoría y filosofía de la historia. Es becario del CONACYT de México y ha sido profesor en varias universidades. Actualmente realiza un estancia de investigación posdoctoral, bajo la dirección de Miguel Ángel Granada, en la Universitat de Barcelona investigando sobre el problema del espacio en la obra de Johannes Kepler. Sus últimas publicaciones son «Gaos -O’Gorman -Ortega y Medina: La construcción de la fenomenología del concepto en la escuela histórica mexicana», Eikasia, 68; «Sobre el concepto de historicidad desde una fenomenología histórica», Historiografías, 10; $\mathrm{y}$ «Tres argumentos keplerianos contra el universo homogéneo», Intus-Legere, 8-2. 


\section{Arena comparada con el firmamento}

El análisis ordinario trata de las magnitudes finitas, en cambio éste penetra hasta el infinito mismo... De tal manera que no sólo abarca el infinito, sino el infinito del infinito, o una infinidad de infinitos. Sólo un análisis de esta naturaleza podría conducirnos hasta los verdaderos principios de las líneas curvas.

L’Hôpital, «preface»²

Ce qui fournit autant qu'on veut de degrés d'incomparables, puisque ce qui est incomparablement plus petit, entre inutilement plus grand que luy, c'est ainsi qu'une parcelle de la matiere magnetique qui passe à travers du verre n'est pas comparable avec un grain de sable, ny ce grain avec le globe de la terre, ny ce globe avec le firmament.

Leibniz, Carta a Varignon, 2/II/1702 3

En octubre de 1684 apareció un pequeño artículo de sólo seis páginas en el Acta Eroditorum de Leipzig de la pluma de Wilhelm Leibniz. Lo sucinto del mismo, la falta de explicaciones, la oscuridad del tema y en general todos los defectos de la comunicación escrita en latín del autor hicieron casi incomprensible su significado. Sin embargo, en sus páginas contenía la primera declaración abierta y explícita de que la larga búsqueda por cuadrar lo curvo, que los matemáticos habían emprendido desde la época de los griegos, y el problema de trazar tangentes, eran ambos en realidad dos aspectos de la misma cosa. El problema de la cuadratura del círculo, que ampliado es el problema de la relación que mantiene toda curva con cada punto en su circunferencia, quedaba comprendido por un nuevo análisis que abría las puertas a su resolución de forma inmediata; y la llave de ese análisis estaba contenida en una observación en apariencia sencilla, la cual en 1692 el marqués de L’Hôpital (1661-1704) lo resumió así:

... las curvas al ser poligonales de una infinidad de lados [côtés], y al diferir entre ellas sólo por la diferencia [différence] de los ángulos que estos lados infinitamente pequeños forman entre sí, al análisis de los infinitamente pequeños únicamente corresponde determinar la posición de estos lados para obtener la curva que de ellos forman, es decir, las

[2] L'Hôpital: Análisis de los infinitamente pequeños para el estudio de las líneas curvas. México: UNAM, 1998, p. 15.

[3] Leibniz, G. W.: Die Matematischen Schriften. Berlin: Weidmannsche Buchhandlung, 1849-1863, vol. IV, p. 91.

Thémata. Revista de Filosofía $\mathrm{N}^{\circ} 53$ (2016) pp.: 13-30. 
tangentes de estas curvas, sus perpendiculares, sus puntos de inflexión o de retorno, los rayos que se reflejan, los que rompen, etcétera. ${ }^{4}$

La descripción de L'Hôpital resume muy bien de dónde partía el nuevo análisis infinitesimal. Primero, las curvas se consideran como polígonos de infinitos lados. Segundo, cada uno de esos lados infinitesimales es matemáticamente diferente de otros y puede ser ubicado. Tercero, como cada punto está plenamente identificado entonces se pueden determinar los más importantes, como los puntos de inflexión o los máximos y mínimos, junto con sus rectas tangentes. Finalmente, como cada infinitesimal es diferente y está determinado por su posición en la curva, desde cualquier punto se puede volver a trazar la curva completa, aunque con algunas reservas, lo cual implica que también es posible encontrar su superficie, volumen y perímetro. Todo esto se resume en el teorema fundamental del nuevo cálculo: los problemas de tangentes y de cuadraturas son equivalentes e inversos.

Sin embargo, el pequeño artículo al que nos referimos, titulado Nova methodus pro maximis et minimis, itemque tangentibus, quae nec irrationales quantitates moratur, et singulare pro illis calculi gemus ${ }^{5}$ (1684), no ponía las cosas tan fáciles. El Nova methodus, como mejor se le conoce, comienza directamente describiendo el algoritmo de cálculo de lo que ahora llamamos derivadas (llamadas «diferencias» por L'Hôpital). El escrito arranca con las operaciones de infinitesimales sin ofrecer definiciones, comentarios o siquiera una introducción general. Lo anterior da una muestra del grado de abstracción que manejó Leibniz y explica las dificultades que sus contemporáneos tuvieron para entender lo que exponía. Por lo tanto, cuando el filósofo alemán afirmó que «la demostración de todo será muy fácil para uno versado en estas materias» ${ }^{6}$ era demasiado optimista cuando menos. La famosa frase de los Bernoulli lo representa mejor, el trabajo de Leibniz era "un énigme plutôt qu'une explication.»

Las operaciones que enseña a realizar el Nova methodus eran las cuatro clásicas de la aritmética: sumar, restar multiplicar y dividir, sin embargo todas adquieren un nuevo significado al operar con infinitesimales en lugar de números. Las operaciones se aplicaban ahora a indivisibles o diferencias, que expresan la medida de cambio entre dos puntos de una

\footnotetext{
[4] L'Hôpital: op. cit., p. 15.
}

[5] «Un nuevo método para los máximos y los mínimos, así como para las tangentes, que no se detiene ante las cantidades fraccionarias o irracionales y es un singular género de cálculo para estos problemas» contenido en Leibniz, G. W.: Die Matematischen Schriften cit., vol. V, p. 220-226.

[6] Leibniz, G. W.: Análisis infinitesimal cit., p. 9.

Thémata. Revista de Filosofía $\mathrm{N}^{\circ} 53$ (2016) pp.: 13-30. 
curva. Este escrito se puede definir como una exposición con un enfoque operativo, donde se enseña el manejo del nuevo algoritmo para calcular con infinitesimales y se muestra un sistema de notación para dicho propósito. Además también se introduce una nueva simbología, que en sí misma fue un avance significativo, introduciendo el símbolo «d» para denotar la diferenciación como algo ya construido ${ }^{7}$.

La verdadera carta fuerte de presentación del nuevo cálculo fue la solución de una serie de complicados problemas que atormentaban a los geómetras analíticos de esa época. Al ir desarrollando la explicación, uno a uno los grandes problemas de la geometría de curvas van cayendo. Con cada nueva interpretación geométrica de los datos, Leibniz hilvana una nueva solución: la transformación del valor de una diferencia indica la presencia de un máximo o un mínimo, según sea de positivo a negativo o viceversa; si la diferencia del eje ordenado y de las abscisas es infinita entonces la tangente es perpendicular al eje de las abscisas, mientras que si los infinitesimales son iguales, entonces la tangente es paralela al eje de las ordenadas, etc. Gracias a ello, Leibniz declaró con orgullo:

Del conocimiento de este Algoritmo, así lo llamo, o de este cálculo, que llamo diferencial, puede obtenerse todas las otras ecuaciones diferenciales por medio del álgebra [calculum] común, y los máximos y mínimos, así como pueden obtenerse las tangentes, de tal forma que no sea necesario separar fracciones o los irracionales u otros vínculos, como sin embargo, debía hacerse según los métodos hasta ahora publicados. $^{8}$

El gran logro del nuevo algoritmo era precisamente que unificaba todos los problemas que estaban en ese momento dispersos. No es de extrañar que los problemas geométricos sean los primeros en ser atacados por Leibniz, ya que el nuevo análisis de infinitesimales se fincaba sobre el análisis espacioso de Vieta, Descartes y Fermat, conservando mucho de su sabor geométrico. Incluso en el pequeño Nova methodus, tan parco en demostraciones, se habla de que las diferencias se pueden considerar como una «cuarta proporcional», adelantando un poco que la demostración del

[7] La simbología leibniziana del cálculo fue un mérito que lo diferenció del de Newton, mérito negado por largo tiempo luego de la polémica con los matemáticos ingleses. Como afirma Oscar Esquisabel: «El diseño leibniziano del cálculo infinitesimal puede ser considerado como parte de su proyecto de una característica general, entendida esta última en el sentido de un arte o disciplina que tiene como meta fundamental el diseño de sistemas simbólicos eficientes para todas las ciencias» Esquisabel, O.: «Infinitesimales y conocimiento simbólico en Leibniz» en Notae Philosophicae Scientiae Formalis 1, n.1, 2012, p. 67.

[8] Leibniz, G. W.: Análisis infinitesimal cit. p. 8.

Thémata. Revista de Filosofía №53 (2016) pp.: 13-30. 
nuevo algoritmo está construida sobre una tradición matemática que se remonta hasta Grecia.

Sin embargo, esa misma tradición aparece literalmente modernizada por los trabajos de geometría analítica, pues las curvas están situadas sobre ejes de referencia y son expresadas por ecuaciones; congregando de esa forma el mundo algebraico y el geométrico. Ahora, con el nuevo análisis infinitesimal, Leibniz pretendía llevar esa tradición un paso más lejos. El filósofo alemán escribió: "Además no me parece que añado en vano que la Geometría con este método es llevada mucho más allá de los límites propuestos por Vieta y Descartes ${ }^{9}$.

Pero si la tradición geométrica-aritmética de los franceses era la que daba cuerpo y concreción a las ideas de Leibniz, la inspiración de esas ideas estaba en el arte combinatorio. Desde la cábala de Llull y el arte de la memoria de Giordano Bruno, la combinatoria sirvió como la fuente de inspiración del pensamiento leibniziano. Una combinatoria de las diferencias o, más radical quizás, de los contrarios, desde la cual brotaba la unidad dialéctica entre opuestos. En ese sentido, Leibniz se inserta en la tradición de pensamiento pitagórico-platónica que puede ser rastreada desde la teología negativa medieval hasta los filósofos florentinos, como explica Bernardino Orio:

\footnotetext{
Heredero de una vieja idea que se remonta a los llamados "prisci theologi» y que reciben Platón, Aristóteles y Plotino, en una formidable tradición que, atravesando la Edad Media tanto judía o musulmana como cristiana, llega hasta la Escuela de Florencia de Ficino y Pico della Mirandola y los neoplatónicos naturalistas del Renacimiento, Cardano, Cusa o Campanella, Leibniz había concebido un mundo unitario, orgánico, activo, energético, un vasto sistema de sistemas arquitectónicos, nunca clausurado por sus datos en origen sino abierto a infinitas perspectivas irreversibles. ${ }^{10}$
}

El nuevo algoritmo infinitesimal no fue posible sin la convicción de Leibniz, como antes la de Bruno, de que el universo funciona como una serie de combinaciones dialécticas que se despliegan desde el Uno trascendente (Monas monadum). La tesis central del cálculo es que las diferencias y las integraciones ("sumas» en terminología leibniziana) son opuestas y también complementarias, lo cual no es más que una forma concreta del presupuesto unitario primero. En ese sentido, diferenciar e integrar no son dos operaciones aisladas, sino la misma en sentidos inversos. Esta convicción la afirmó con claridad Leibniz en un escrito algo posterior: «Pues como

[9] Leibniz, G. W.: Análisis infinitesimal cit. p. 23.

[10] Orio, B.: «Leibniz y la tradición hermética» en THÉMATA. Revista de filosofía 42, 2009, p. 109.

Thémata. Revista de Filosofía $\mathrm{N}^{\circ} 53$ (2016) pp.: 13-30. 
las potencias y las raíces en los cálculos comunes [vulgaribus calculis], las sumas y las diferencias o «\》 y «d» son recíprocas [reciprocae]»" ${ }^{11}$. Esa idea fructífera de unidad de suma/sustracción, aumento/disminución, existía de forma previa al cálculo de infinitesimales. Javier de Lorenzo la descubre ya en el De corpore (1655) de Hobbes, aunque afirma también que existía incluso desde antes:

\footnotetext{
Dos operaciones, suma y sustracción, claves para la combinatoria de nuestra razón, para el cálculo en que consiste el razonamiento. Idea no sólo de Hobbes, ciertamente, sino que estaba en el ambiente, en la búsqueda de una compensación a la Torre de Babel en que se había visto sometida la especie humana. Idea que también explicitó Descartes cuya carta del 20 de Noviembre de 1629 a Mersenne copia Leibniz hacia 1678. Copia a la que Leibniz agrega que la invención de una lengua o al menos una escritura para expresar los pensamientos sería algo maravilloso sobre todo para acabar las disputas que dependen del razonamiento; porque con esa lengua o escritura razonar y calcular serían lo mismo. ${ }^{12}$
}

La combinatoria y la búsqueda de un lenguaje universal son parte de una misma forma de razonar, de ahí que Leibniz se interesó tanto por la lógica pura como por la creación de un simbolismo adecuado para las matemáticas. Ambas cosas las concibió para superar las ambigüedades y expresar de una forma más precisa los vínculos de la realidad. Pero existe una dificultad en la aplicación de la combinatoria de opuestos en el cálculo, pues la combinatoria es un arte esencialmente discreto y al igual que las formas numéricas trabaja con unidades cerradas, y en última instancia se acerca más a las realidades lingüísticas. En cambio, los objetos de la geometría, donde el cálculo tenía su aplicación más inmediata, existen de manera continua. Las mismas líneas carecen de partes y más bien fluyen siempre como un todo. Sólo los puntos se acercan a lo discreto, pero el mismo Leibniz no creyó siquiera que éstos fueran parte de la línea. Así lo afirmó en varios lugares: «los puntos hablando con exactitud, son extremidades de la extensión y de ningún modo partes constitutivas de las cosas: la geometría lo muestra. ${ }^{13}$

Se requería entonces de un engarce que existiera entre los dos extremos, algo que hiciera posible pasar de lo discreto a lo continuo. Leibniz

[11] Leibniz, G. W.: Análisis infinitesimal cit., p. 25.

[12] Lorenzo, J. [en línea]: «Leibniz-L’Hôpital y el cálculo diferencial» en La ciencia europea de 1650 hasta 1800. Canarias: Encuentros Educativos, Canarias, 2007, http://www.gobcan. es/educacion/3/usrn/fundoro/web_fcohc/005_publicaciones/seminario/ciencia_europea2.htm [Consultado: 30/08/2014].

[13] Leibniz a Samel Masson, 1716: Tomo la traducción de Luna, M.: La ley de la continuidad en G. W. Leibniz. Sevilla: Universidad de Sevilla, 1996, p. 158. Cfr: Respuesta de Foucher a Leibniz sobre su nuevo sistema del conocimiento de las sustancias.

Thémata. Revista de Filosofía $\mathrm{N}^{\circ} 53$ (2016) pp.: 13-30. 
necesitaba algo que mediara entre las sumas y las restas de elementos discretos y los puntos y las líneas de lo geométrico. Este engarce lo encontró en la noción de infinitésimo, tomada de Wallis vía Huygens, pero desarrollada para intentar superar las ambigüedades de la terminología que se venía arrastrando desde los indivisibles. El infinitésimo es la diferencia entre dos puntos de una recta, separados por una distancia tan pequeña que el arco de la curva coincide con la tangente. Esta definición paradójica resume bien el extraño ente que «no siendo un número concreto participa evanescentemente de lo numérico.» ${ }^{14}$ Pues la esencia del infinitésimo es que es menor que cualquier número, pero sin caer en la nada.

Sin embargo, esta unión de lo paradójico era por su misma naturaleza de difícil explicación. En el Nova methodus, sin utilizar propiamente el término «infinitesimal», Leibniz explica su uso de la siguiente manera:

En esta situación se consigue, para encontrar la tangente, trazar la recta que una dos puntos de una curva que estén a una distancia infinitamente pequeña o el lado prolongado de un polígono de infinitos ángulos, que para nosotros equivale a la curva. Esta distancia infinitamente pequeña siempre puede ser expresada por alguna diferencial conocida... ${ }^{15}$

Si se sigue este razonamiento, una curva es al mismo tiempo un trazo continuo y también un polígono de infinitos lados. La paradoja se resuelve usando a esos extraños entes que oscilaban entre el ser y la nada. Sin embargo, ese delicado equilibrio se altera apenas uno se decanta por cualquiera de las opciones. Si los infinitesimales son reales, entonces existe al mismo tiempo lo finito y lo infinito dentro del mismo cálculo, pero si no son reales, entonces ni siquiera alcanzan para realizar el menor cálculo.

Para expresar esto último con términos matemáticos, podemos decir que para que la operación con infinitesimales sea válida, la tangente y la secante deben ser idénticas. Eso implica que la distancia entre dos puntos sea otro punto, pero no existe una proporción entre puntos y líneas, excepto si, como dice Aristóteles, "la línea sea un compuesto de puntos» (Fis. 215b.), cuestión que ya había rechazado Leibniz. Pero incluso si se aceptara esa opción, eso conllevaría la existencia de un infinito actual y de

[14] Esta idea viola claramente los principios de la lógica aristotélica. Pues dice el libro de la Física: «el vacío no tiene razón ( (X́Yov) por la cual fuera superada por un cuerpo, como tampoco la nada la tiene con el número.» Fis. 215b. 12-14 [Schmidt, 2001: 88]. La idea del cálculo, sin embargo, da pie a proyectar un valor cuya magnitud es menor que cualquier razón dada de antemano. Una definición de Leibniz en Theoria motus abstracti de punto afirma: "Punctum non est, cujus pars nulla est, nec cujus partes sunt indistantes, cujus magnitudo est inconsiderabilis, inassignabilis, minor quam quae ratione» Leibniz, G. W.: Die Philosophischen Schriften. Berlín: Weidmannsche Buchhandlung, 1880 vol. IV-II p. 229.

[15] Leibniz, G. W.: Análisis infinitesimal cit., p. 9.

Thémata. Revista de Filosofía $\mathrm{N}^{\circ} 53$ (2016) pp.: 13-30. 
magnitudes sin valor pero reales. Ante esta complicada discusión, Leibniz siempre trató de alejar su nuevo cálculo del debate metafísico:

mi propósito fue señalar que no hay necesidad de hacer depender el análisis matemático de las controversias metafísicas ni de asegurar que haya en la naturaleza estrictamente líneas infinitamente pequeñas, en comparación con las nuestras, ni por consiguiente haya líneas infinitamente más grandes que las nuestras. [...] Por lo cual, a fin de evitar estas sutilezas, he creído que para hacer sensible a todo el mundo el razonamiento, era suficiente con explicar aquí el infinito por los incomparables, es decir, concebir cantidades incomparablemente más grandes o más pequeñas que las nuestras; esto nos proporciona todo lo que necesitamos en cuanto a grados de incomparables.... ${ }^{16}$

Esa sana intención falló. Después de publicar su algoritmo, la comunidad de matemáticos comenzó a discutir sobre si se debía considerar a los infinitésimos como entidades reales o no. El mismo Leibniz tuvo un largo comercio epistolar con Johann Bernoulli, debatiendo precisamente este asunto, debido a una implicación mal deducida por el matemático suizo ${ }^{17}$. Para evitar desde malentendidos hasta los sensibles problemas de lógica interna, Leibniz calificará insistentemente a los infinitesimales de "fictions, mais des fictions utiles.» El problema de fondo, la proporción entre lo finito y lo infinito, no era en sí mismo nada nuevo, ya desde los griegos aparecía siempre que se intentaba construir proporciones entre líneas y arcos. Cada vez que esto ocurrió, parecía que los matemáticos debían optar entre la funcionalidad de los métodos y el rigor que exigía la demostración. Para salvar el rigor, Leibniz se esforzó en conservar el carácter continuo de las líneas y, por ello, se enfrentó a la virtualidad de los infinitesimales, aunque no por eso afirme su inexistencia. Como escribe Javier de Lorenzo:

Para un Pascal, para un Leibniz, el infinitésimo es un elemento que impone la razón conceptual, como el punto en el infinito, aunque no lo capte el entendimiento. Y lo que puede hacerse es dar una justificación y explicar unas reglas de manejo de tales nuevas magnitudes y en su enlace con las antiguas. Pascal dio esa justificación mediante el recíproco del postulado eudoxiano-arquimediano, y Leibniz acepta dicha justificación según se desprende de algunas cartas, a la vez que formula las reglas de manejo de las diferencias. ${ }^{18}$

Pero si el infinitésimo está por encima del entendimiento, entonces está aún más allá de la percepción sensitiva. Sin embargo, parte de

[16] Leibniz, G. W.: Die Matematischen Schriften cit., vol. IV, p. 91.

[17] Montesinos hace la reseña completa de dicha discusión: Montesinos, J.: «Fluxiones, infinitesimales y fuerzas vivas. Un panorama leibniziano» en THÉMATA. Revista de filosofía 42, 2009, p. 77-106.

[18] Javier de Lorenzo, «Estudio preliminar» en Leibniz, G. W.: Análisis infinitesimal cit., p. lxviii.

Thémata. Revista de Filosofía №53 (2016) pp.: 13-30. 
la confusión que originó el nuevo cálculo fue que quienes lo aprendían, literalmente creían ver como se construía un infinitesimal. Esto es claro en el tratado De geometria recondita et analysi indivisibilium atque infinitorum aparecido en el Acta Eroditorum en $1686^{19}$. Ahí Leibniz dio algunas explicaciones más acerca del nuevo análisis y ofreció un esbozo de la demostración que depende del uso del llamado triángulo característico que proviene desde Pascal. El problema de usar dicho triángulo era que da la impresión de poder verlo cuando se traza el diagrama. Probablemente por eso Leibniz en De geometria no incluyó ningún diagrama, pero la explicación e incluso el mismo nombre de triángulo condiciona al lector a formarse una imagen del mismo.

Todo lo anterior podría aclararnos porqué Leibniz se centró, en el Nova methodus, en ofrecer ejemplos de aplicación. Esos ejemplos mostraban la potencia y alcance de las nuevas matemáticas, resolviendo problemas difíciles de manera casi inmediata, sin complicarse demasiado justificando la difícil paradoja combinatoria en que estaba construido el cálculo. Precisamente dicha potencia del nuevo cálculo era algo de lo cual Leibniz se sentía sumamente orgulloso, y siempre resaltó que éste no tenía que limitarse a cantidades algebraicas, sino que abarcaba por igual «fracciones e irracionales que componen a las variables» y "también es evidente que nuestro método se extiende a las líneas trascendentes, que no pueden ser manejadas por el cálculo algebraico.» Al resolver nuevos problemas, el cálculo amplió el campo de las matemáticas hacia nuevos límites, confirmando con ello la fe leibniziana en la unidad subyacente a todo lo real. Como él mismo declara:

Y ciertamente estos puntos son los inicios de una Geometría muy sublime que también se extiende a los dificilísimos y hermosos problemas de la Matemática mixta que sin nuestro cálculo diferencial o semejante nadie podrá tratar con parecida facilidad..$^{20}$

[19] En español: «Sobre una geometría altamente oculta y el análisis de los indivisibles y de los infinitos». Leibniz, G. W.: Die Matematischen Schriften cit., vol. V, pp. 226-233.

[20] Leibniz, G. W.: Análisis infinitesimal cit., p. 14.b,mn

Thémata. Revista de Filosofía $\mathrm{N}^{\circ} 53$ (2016) pp.: 13-30. 


\section{Entre luz y tinieblas}

Video plerosque, qui Mathematicis doctrinis delectantur, a Metaphysicis abhorrere, quo in illis lucem, in his tenebras animadvertant.

Leibniz, De primae philosophiae Emendatione, et de Notione Substantiae ${ }^{21}$

Los fundamentos del cálculo de infinitesimales le sirvieron a Leibniz para unificar, en materias muy diversas, otras ideas también en apariencia opuestas, como la extensión y la energía en metafísica, o la pasivo y lo activo en dinámica. Gracias al ejemplo del cálculo, esas contradicciones podían ser explicadas como elementos recíprocos, igual que se había demostrado que eran lo discreto y lo continuo o el punto y la línea. El pensamiento leibniziano se esfuerza por encontrar puentes entre áreas extremas y reconciliarlas. En política su aspiración más grande fue reconciliar a católicos y protestantes, en filosofía deseaba unificar a los antiguos con los modernos. En ambos casos él creía que existían verdades profundas y que cada grupo podía tener parte de las mismas. Es por ello que decide rehabilitar el concepto aristotélico de entelechia o forma sustancial y aplicarlo a su análisis de la materia.

La decisión de Leibniz de establecer una identidad entre la actividad más íntima de la materia y las formas sustanciales dejó entrar a las explicaciones basadas en las causas finales dentro del mecanicismo del siglo XVII. Gracias a ello, el universo se podía pensar tanto como el resultado de la velocidad, trayectoria y choques de los cuerpos, y también como un cosmos, donde los principios de perfección, armonía y bondad juegan un papel preponderante ${ }^{22}$. Con esas bases, Leibniz podía otorgar un lugar a las ideas renacentistas sobre las almas, inteligencias y fuerzas que existían en la materia, la cual ya no se debía concebir sólo como algo meramente cuantitativo. Además al dotar a los cuerpos de características propias los individualizaba. Sin embargo, el hilo conductor de los descubrimientos matemáticos y físicos de Leibniz, la idea de continuo, le cerraba en metafí-

[21] Leibniz, G. W.: Die philosophische Schrifen cit., vol. IV, p. 468.

[22] Leibniz enlaza la idea de armonía con la existencia de un ser eterno, infinito y pensante, y por ende niega a la mera materia que sea eterna y pensante: «De esta manera que si la materia fuese el primer ser pensante y eterno, habría un número infinito de seres eternos, infinitos y pensantes, que serían independientes unos de otros, cuyas fuerzas serían limitadas y sus pensamientos distintos, y por consiguiente jamás podrían producir este orden, esta armonía y esta belleza que se observa en la naturaleza.» Leibniz, G. W.: Nuevos ensayos sobre el entendimiento humano. Madrid: Alianza, 1992, p. 527 en adelante se citará por la notación canónica: N.E. IV, X.

Thémata. Revista de Filosofía №53 (2016) pp.: 13-30. 
sica el paso para integrar a los individuos plenos, cuya realidad paradójicamente siempre fue considerada por él como un dato fundamental. ${ }^{23}$

El principio de continuidad exigía que no se dieran ningún tipo de saltos: la materia debía ser infinitamente divisible, porque si existieran átomos, entonces habría fragmentos en la materia cualitativamente distintos del conjunto. Tampoco, en términos estrictos, podrían existir individuos separados de su entorno, ni almas particulares, pues ambas cosas implican un salto entre el individuo y lo que no es el individuo. Y si llevamos el principio de continuidad a sus últimas consecuencias, tampoco existiría la multiplicidad y sólo quedaría el uno-infinito. "Sin embargo, si no hubiese verdaderas unidades sustanciales, no habría nada sustancial ni real en la colección.»

Esa consideración lo llevaba a creer que existían los átomos y las unidades reales, no obstante, como refiere el mismo Leibniz:

...había dado en el vacío y en los átomos, pues esto es lo que satisface mejor la imaginación, pero habiendo regresado de allí, después de muchas meditaciones, advertí que es imposible encontrar los principios de una verdadera unidad en la materia sola, es decir, en lo que es sólo pasivo, puesto que el todo no es más que una colección o amontonamiento de partes hasta el infinito. ${ }^{24}$

Para resumir, Leibniz necesitaba que existieran átomos para que existieran verdaderas unidades, pero esos átomos no podían ser materiales. Intentando resolver esa contradicción, Leibniz dio el paso desde una explicación física a una metafísica. Adentrándose así en lo que llamaba el laberinto del continuo, pues aunque «un geómetra no tiene necesidad de turbarse el espíritu con el famoso laberinto de la composición del continuo... puesto que el geómetra puede concluir todas sus demostraciones [...] sin entrar en estas discusiones, las cuales no dejan de ser necesarias e importantes en filosofía y en la teología. $»^{25}$

Como en el continuo los entes no son más reales que el todo, sino compuestos transitorios, Leibniz comprendió que se necesitan unidades

[23] Desde el escrito de Leibniz: Disputatio metaphysica de principio individui, que elabora en 1663 para obtener el grado de bachiller en filosofía, ya muestra las preocupaciones del joven sajón por definir con claridad la individualidad, afirmando que: «Todo individuo es individualizado por su entidad total» Leibniz, G. W.: Discusión metafísica sobre el principio de individuación. México: UNAM, 2009, p. 17.

[24] Leibniz, G. W.: Tratados fundamentales cit., p. 11.

[25] Leibniz, G. W.: Tratados fundamentales cit., p. 117-118.

Thémata. Revista de Filosofía №53 (2016) pp.: 13-30. 
auténticas para dar soporte a las sustancias. Debían por lo tanto postularse los átomos, pero no pensarlos como materiales:

[Porque] los átomos de materia son contrarios a la razón, además de estar compuestos de partes, puesto que el enlace indivisible de una parte con otra (si pudiera concebirse o suponerse razonablemente) no destruiría su diversidad. Sólo los átomos de sustancia [Atomes de substance], es decir, las unidades reales y absolutamente desprovistas de partes, son las fuentes de las acciones y los primeros principios absolutos de la composición de las cosas y como los últimos elementos del análisis de las sustancias. $^{26}$

La salida al problema que encuentra el filósofo sajón fue postular átomos no materiales a los que llamó puntos metafísicos. Para darle forma a esa idea, Leibniz recurrió de nueva cuenta al ejemplo del cálculo matemático, aplicando la misma lógica combinatoria para darle sentido a sus átomos que, sin ser materiales, son el fundamento de la materia. Pero la unión entre puntos metafísicos y el cálculo fue más allá de esto. Como antes hiciera Giordano Bruno, Leibniz hizo un correlato entre los átomos físicos y los puntos matemáticos. El filósofo alemán afirmó que «los puntos matemáticos son su punto de vista [de los puntos metafísicos] para expresar el universo.» Esta relación es doblemente significativa, pues Leibniz afirmó que Dios creó el mundo como una especie de cálculo combinatorio, donde los puntos son las almas y el continuo era el universo entero; además el alemán señaló que esas almas individuales expresan el universo entero desde su punto de vista. De esa forma, la relación alma/universo quedó establecida como una dialéctica de contrarios.

Para decirlo de forma compacta, Leibniz creó un vínculo dialéctico entre el mínimo y el máximo, entre las unidades particulares y la unidad de unidades. Ese vínculo funcionaba de forma análoga al establecido por el cálculo entre el punto y la línea, entre lo unitario y lo continuo. Con la lógica combinatoria fue posible pasar desde el continuo, que el filósofo alemán considera como real universal, a lo real particular, que son los verdaderos individuos [unités reelles]. En ese sentido el funcionamiento del cálculo y de los puntos metafísicos es casi idéntico, ambos pueden pasar de lo mínimo hacia lo continuo. Sin embargo, el planteamiento establecido en matemáticas se invierte en la metafísica, porque si en el cálculo matemático los infinitésimos tienen sólo una existencia virtual, cuando se habla de sustancia los individuos representan la realidad más auténtica y plena, pues de ellos surge todo lo demás.

Esto se ve claramente reflejado por el rechazo de Leibniz a la idea de un alma universal ${ }^{27}$. En lugar de explicar el universo a partir de un prin-

[26] Leibniz, G. W.: Tratados fundamentales cit., p. 17.

[27] "J'ai montré cy dessus que la pretendue Demostration des Peripateticiens qui soutenoient, qu'il n'y avoit qu'un esprit commun à tous les hommes, est de nulle force et n'est appuyée que

Thémata. Revista de Filosofía №53 (2016) pp.: 13-30. 
cipio único, el alemán optó por considerar la individualidad como algo real, más auténtico que los géneros universales ${ }^{28}$. Con ello, Leibniz se adentra en las polémicas sobre el origen de las almas individuales, teniendo que optar, en apariencia, entre un creacionismo ad hoc o una transmigración constante de las almas. En lugar de optar por alguno, Leibniz prefirió argumentar en favor de la eternidad de toda alma individual. Sobre esto, el sajón escribió: "Les experiences de nostre temps nous portent à croire, que les ames et même les animaux ont tousjours existé." ${ }^{29}$ Pues si todo individuo es una alma y toda alma es eterna, esto implica que en el cosmos no existe ni nacimiento ni muerte, salvo en un sentido aparente. En lugar de existir destrucción y creación, sólo habría aumentos [augmentation], desde lo mínimo hasta el pleno efecto, y reducciones hasta la mínima expresión.

En este línea de pensamientos, Leibniz puso la particularidad de cada ser en un punto, en un átomo indivisible e indestructible que existe desde la eternidad (o desde la creación) y que continuará existiendo indefinidamente. Eso es lo que Leibniz llama mónada y equivale a los puntos metafísicos, pues sólo en ellos existe una verdadera unidad e indivisibilidad. En la Monadología (1714), Leibniz escribió: "La Monade, dont nous parlerons ici, n'est autre chose qu'une substance simple, qui entre dans les composés; simple, c'est-à-dire sans parties. $\|^{30}$

Con la idea de mónada, Leibniz concreta su propia versión del triplici minimo bruniano, ordenando en tres niveles: físico, matemático y metafísico ${ }^{31}$. A estos tres niveles, le corresponde una clase de mínimo: los átomos en física, los puntos en matemáticas y las mónadas en metafísica. Como explicó él mismo:

sur des fausses suppositions.» Leibniz, Considerations sur la doctrine d'un Esprit Universel Unique (1702) en Leibniz, G. W.: Die philosophische Schrifen cit., vol. VI, p. 531.

[28] La individualidad de Leibniz es radical, ninguna cosa o evento se repite en el orden del tiempo y el espacio. Como dice Dilthey, el principio de individualidad prepara la concepción histórica del mundo, pues la riqueza óntica de los acontecimientos es que ninguno es igual a ningún otro: «El rígido sistema natural se cambia de tal modo que Leibniz prepara la concepción histórica del mundo.» Dilthey, Hombre y mundo en los siglos XVI y XVII. México: FCE, 1978, p. 465.

[29] Leibniz, G. W.: Die philosophische Schrifen cit., vol. VI, p. 532.

[30] Leibniz, Monadologie, §1.

[31] La mónada de Leibniz, concepto pitagórico, es un paso significativo para ir más allá del dualismo pera a tal grado que Coudert habla de evolución: "Leibniz's substitution of "force" for "mind" as the defining characteristic of substance represented an evolution in his thinking that ultimately banished the last traces of Cartesian dualism from his system and led to the concept of monads." Coudert, R. S.: Leibniz and the Kabbalah. Dordrecht: Kluwer, 1995, p. 78.

Thémata. Revista de Filosofía ํ53 (2016) pp.: 13-30. 
Así, los puntos físicos sólo son indivisibles en apariencia; los puntos matemáticos son exactos, pero sólo constituyen modalidades: únicamente los puntos metafísicos o de substancia (constituidos por formas o almas) son exactos y reales; y sin ellos no habría nada real, puesto que, sin verdaderas unidades, no habría multiplicidad. ${ }^{32}$

Otra vez la división es sólo una apariencia, pues los tres órdenes guardan vínculos entre sí. Aunque en principio Leibniz no concibe átomos físicos debido a que el principio de continuidad exige que la materia sea infinitamente indivisible, el análisis meramente formal de la matemática había descubierto la conexión entre lo continuo y lo discreto, e hizo posible establecer un vínculo entre ellos. Gracias a ese vínculo, se podían postular átomos verdaderos pero en el ámbito metafísico, que pasa a ser el reino de los entes individuales. Dada la preeminencia de los individuos, el paso anterior implica un cambio de fondo en todo el planteamiento, porque si la actividad íntima de los entes individuales es lo que crea la realidad, entonces lo continuo y la materia depende de la expresión de los individuos. De tal modo que la realidad debe ser continua y discreta a la vez:

Et l'auteur de la nature a pu pratiquer cet artifice divin et infiniment merveilleux, parce que chaque portion de la matière n'est pas seulement divisible à l'infini, comme les anciens ont reconnu, mais encore sous divisée actuellement sans fin, chaque partie en parties, dont chacune a quelque mouvement propre,autrement il serait impossible que chaque portion de la matière pût exprimer tout l'univers. ${ }^{33}$

La esencia de los individuos es la fuerza, concepto que para Leibniz representa la expresión de las almas individuales o mónadas, ${ }^{34}$ las cuales como vimos debían ser absolutamente únicas y particulares. Pero para que éstas fueran verdaderas unidades era necesario que carecieran de partes, pues lógicamente sólo lo que tiene partes puede descomponerse. Sin embargo, cuando algo no tiene partes, tampoco puede tener contacto con otra cosa; como aclara Leibniz: «Or, là où il n'y a point de parties, il n'y a ni étendue, ni figure, ni divisibilité possible» $^{35}$. Esta falta de partes era un requisito para que las mónadas fueran verdaderamente eternas, pero esta misma propiedad hacía que las mónadas estuvieran por completo aisladas unas de otras. La famosa frase de Leibniz: "Les

[32] Leibniz, G. W.: Tratados fundamentales cit., p. 18.

[33] Leibniz, Monadologie, §65.

[34] En rigor, ambas cosas no son por completo equivalentes, pues mónada es toda sustancia individual, alma toda sustancia individual con pensamiento, y espíritu se reserva para los seres racionales. Leibniz, Dircours, §XXXIV. Cfr. Leibniz, Monadologie, §19.

[35] Leibniz, Monadologie, §3.

Thémata. Revista de Filosofía $\mathrm{N}^{\circ} 53$ (2016) pp.: 13-30. 
monades n'ont point de fenêtres", resume mejor que muchas otras el sentido del solipsismo radical que la tesis de la individualidad absoluta acarrea.

Aunque en estricto sentido las mónadas están cerradas al exterior y sólo conocen su propia actividad interna, desde otra perspectiva son lo más abierto, pues cada mónada porta una imagen completa del universo ${ }^{36}$. Esto incluía ser el reflejo de todos los demás entes individuales, así como de todas las acciones pasadas y futuras. Las mónadas fueron para Leibniz el espejo del infinito [miroir vivant perpétuel de l'univers]:

En efecto, toda sustancia expresa, aunque confusamente, cuanto sucede en el universo, pasado, presente o porvenir, lo cual tiene alguna semejanza con el conocimiento infinito; y como todas las demás sustancias expresan a ésta a su vez, y se conforman a ella, puede decirse que extiende su poder a todas las demás, a imitación de la omnipotencia del creador. ${ }^{37}$

Si bien la fuerza poética de las mónadas leibnizianas como espejos infinitos sirve como crisol de la imaginación, como una salida al dualismo mecanicista no es satisfactoria. Las mónadas como entes metafísicos no se comunican con la materia y sólo parecen influir en el mundo físico debido a la doctrina de la armonía preestablecida que postuló Leibniz. Dicha armonía se concreta con la imagen de los dos relojes, independientes pero eternamente sincronizados, metáfora que no resuelve el problema, sólo hace superflua la solución. Así Dilthey calificó a esto como una "artificiosa y estéril teoría»" ${ }^{38}$. Aunque si nos atenemos a los principios internos del sistema y aplicando una lógica combinatoria no era necesario duplicar el reloj, la salida posible a esta desconexión de lo material y espiritual estaría en su encuentro de ambas en una realidad superior que necesariamente tendría que ser infinita, pero dicha unión apuntaría hacia un panteísmo monista, y al parecer ése era precisamente el problema.

Pese a que Leibniz es famoso por su uso del concepto de infinito, en realidad este fue un concepto problemático dentro de su sistema. Los mónadas que postuló, además de eternas en duración, también son infinitas en número. Aunque en matemáticas el sajón optó por no utilizar el infinito en acto, en el plano metafísico Leibniz rechazó la doctrina de Aristóteles que lo negaba y postuló un todo auténticamente infinito. El universo leibniziano es un continuo pleno, que no sólo es teóricamente divisible al infinito, sino que está infinitamente dividido en acto. Es por lo tanto ilimitado en extensión y en cantidad de

[36] «El carácter individual y autárquico de las mónadas sería absoluto con respecto a los fenómenos, pero con respecto a su creador — que contiene en sí todas las perfecciones de su creación- o incluso de la serie, no hay autonomía total.» Salas Ortuela, J., «Hegel y Leibniz frente a Spinoza» en Logos: Anales del seminario de metafísica 10, 1975, p. 105.

[37] Leibniz, G. W.: Tratados fundamentales cit., p. 116.

[38] Dilthey, Hombre y mundo cit., p. 465.

Thémata. Revista de Filosofía $\mathrm{N}^{\circ} 53$ (2016) pp.: 13-30. 
sustancias infinitesimales ${ }^{39}$, y es también eterno en duración, pues sólo puede perecer por aniquilación. Además es infinitamente infinito, pues cada una de sus partes, cada mónada, cada punto metafísico infinitamente pequeño, refleja el cosmos infinito. Así el universo es siempre infinito de infinitos, la máxima unidad plena posible.

A pesar de esto, el mismo Leibniz fue oscuro respecto a aceptar un universo físico infinito en acto. La misma definición de lo infinito como sólo aplicable al absoluto, que el sajón reservó para Dios, implicó esta separación entre realidad física e infinitud. Así, Leticia Cabañas escribe que la aplicación del infinito al mundo físico es contradictoria con el continuo:

Para superar la contradicción hay que pasar de poner el infinito en el objeto a ponerlo en el espíritu, en una ley ideal que se convierte en el instrumento de investigación por excelencia para penetrar lo real. Los procesos infinitos, que siempre habían sido considerados inalcanzables para el hombre, pueden ser ahora descritos mediante la aplicación del infinito matemático al mundo físico. ${ }^{40}$

La interpretación de Cabañas hace justicia a los esfuerzos conceptuales leibnizianos, pero deja fuera a sus ideas que apuntan hacia un infinito universo físico real. El intento por parte del sajón de evitar cualquier implicación panteísta que refutara sus bases cristianas explica por qué no admitió las consecuencias de sus propios postulados. En realidad la idea de continuo no es contradictoria con un universo físico infinito, salvo que se quiera hacer del prejuicio del realismo materialista un axioma, pues puede insertarse esa idea en el marco dialéctico de la combinatoria y hacer de la dualidad continuo/discreto un eje del despliegue del universo físico. El problema central está, como bien notó Alejandro Herrera, en que Leibniz rechazó aplicar al universo la categoría de «auténtico todo»" ${ }^{41}$ el cual reservó únicamente para su Dios trascendente. El resultado es un mundo físico potencialmente infinito, otro metafísico dividido en acto al infinito y Dios como unidad trascendente absoluta.

En contraste con lo anterior, desde la dialéctica pitagórica era posible postular un universo autónomo absoluto, donde el propio universo

[39] Concuerdo con la tesis de Richard T. W. Arthur cuando afirma que: "The real Enigma, I propose, is that this thesis, that the continuum is not just potentially but actually divided into an infinity of parts, is one he seems to have held from as early as 1666, and consistently from then on, even while proposing atoms." Arthur, Richard T. W., 2003, "The Enigma of Leibniz's Atomism», en Oxford Studies in Early Modern Philosophy 1, 2003, p. 187. En esa idea lo continuo está compuesto de partes infinitesimales, que no pueden ser sólo materiales, únicamente pueden ser materiales-espirituales o monádicas.

[40] Cabañas, L.: «El concepto de infinito en Leibniz y Locke» en Ontology Studies 10, 2010, p. 150.

[41] Herrera, A.: "El infinito en Leibniz" en Benítez L., y Robles J. A. El problema del infinito: filosofía y matemáticas. México: UNAM-IIF, 1997, p. 165.

Thémata. Revista de Filosofía №53 (2016) pp.: 13-30. 
asume la función de reunir los opuestos convirtiéndose en el correlato de la divinidad. En tal caso estaría el infinito en el universo y no fuera del mismo, con lo cual la idea de 'creación' carecería de sentido y también toda diferencia entre universo y absoluto. A pesar de los intentos del propio Leibniz por evitar llegar a la conclusión anterior, todo su sistema apuntaba en esa dirección. Esa consecuencia se desprende del núcleo de su pensamiento, como bien lo notara Samuel Clarke. "Éste, en su respuesta 4a, colocará a Leibniz ante la consecuencia inevitable de su razonamiento: que el mundo material habrá de ser infinito y eterno, lo cual contradiría la decisión creadora de Dios. $\rangle^{42}$ Esta consecuencia no es contradictoria con el sistema mismo, sino con los principios cristianos a los que Leibniz concede la acción creadora de la divinidad. Como escribe Salas Ortuela:

En Leibniz, hay un esfuerzo por salvar la trascendencia de Dios con respecto a las mónadas. Esta trascendencia de Dios sólo se aprecia en la dependencia de las mónadas con respecto a Él, pues las criaturas no son sino realizaciones imperfectas de Dios. En tanto que realizaciones, hay continuidad entre ellas y Dios, pero en tanto que imperfectas, se da por una parte la diferencia y autonomía, y, por otra, la contingencia y limitación. ${ }^{43}$

El punto de rompimiento es la ubicación de la mónada de mónadas como un ente trascendente o inmanente, fuera o dentro del universo. Leibniz trató de limitar la acción divina al cálculo combinatorio primordial que dio origen al cosmos, creando entonces, según su metáfora, dos relojes perfectamente sincronizados, uno físico continuo y otro metafísico discreto, y reservándose un papel en un futuro indefinido donde clausurará sus obras. Pero, aun con su intento de separar a Dios y el universo, la causa y el efecto, el principio y la unidad, la idea de armonía que guía su pensamiento, y la necesidad de caracterizar el cosmos por su perfección infinita impele a considerar dicha división como "une fiction d'esprit.»

\section{Bibliografía}

Arthur, T. W. R.: «The Enigma of Leibniz's Atomism» en Oxford Studies in Early Modern Philosophy 1, 2003, pp. 183-227.

Benítez, L., y Robles J. A.: El problema del infinito: filosofía y matemáticas. México: UNAM-IIF, 1997, p. 165.

[42] Ribas, A.: Biografía del vacio. Barcelona: Editorial Sunya, 2008, p. 250.

[43] Salas, J.: «Hegel y Leibniz frente a Spinoza» cit. p. 106.

Thémata. Revista de Filosofía $\mathrm{N}^{\circ} 53$ (2016) pp.: 13-30. 
Cabañas, L.: «El concepto de infinito en Leibniz y Locke» en Ontology Studies 10, 2010, pp. 143-152.

Coudert, R. S.: Leibniz and the Kabbalah. Dordrecht: Kluwer, 1995, p. 78.

Dilthey, W.: Hombre y mundo en los siglos XXI y XVII. México: FCE, 1978, p. 465.

Esquisabel, O.: «Infinitesimales y conocimiento simbólico en Leibniz» en Notae Philosophicae Scientiae Formalis 1, n.1, 2012, pp. 66-79.

González-Velazco, E.: Journey Through Mathematics, New York: Springer, 2010, p. 361.

Leibniz, G. W.: Análisis infinitesimal. Madrid: Tecnos, 1987, p. 5.

- Die Philosophischen Schriften. Berlín: Weidmannsche Buchhandlung, 1880.

- Die Matematischen Schriften. Berlín:Weidmannsche Buchhandlung, 1849-1863.

- Nuevos ensayos sobre el entendimiento humano. Madrid: Alianza, 1992.

- Tratados fundamentales. Buenos Aires, Losada, 2004.

L'Hôpital: Análisis de los infinitamente pequeños para el estudio de las líneas curvas. México: UNAM, 1998, p. 15.

Lorenzo, J. [en línea]: «Leibniz-L’Hôpital y el cálculo diferencial» en $L a$ cienciaeuropea de 1650 hasta 1800. Canarias: Encuentros Educativos, Canarias, 2007,http://www.gobcan.es/educacion/3/usrn/fundoro/web_fcohc/005_ publicaciones/seminario/ciencia_europea2.htm [Consultado: 30/08/2014].

Luna, M.: La ley de la continuidad en G. W. Leibniz. Sevilla: Universidad de Sevilla, 1996, p. 158.

Merker, C. [en línea]: «La «géometrie calculante» de Pascal» http:// epiphymaths.univ-fcomte.fr/Merker-Geometrie_calculante_de_Pascal.pdf [Colsultado: 28/8/2014].

Montesinos, J.: «Fluxiones, infinitesimales y fuerzas vivas. Un panorama leibniziano» en THÉMATA. Revista de filosofía 42, 2009, p. 77-106.

Orio, B.: «Leibniz y la tradición hermética» en THÉMATA. Revista de filosofía 42, 2009, pp. 109-122.

Ribas, A.: Biografía del vacio. Barcelona: Editorial Sunya, 2008, p. 250.

Salas, J.: "Hegel y Leibniz frente a Spinoza» en Logos: Anales del seminario de metafísica 10, 1975, pp. 101-126.

Thémata. Revista de Filosofía №53 (2016) pp.: 13-30. 